\title{
Symmetric variability of gamma-ray emitting blazars
}

\author{
Kenji Yoshida* \\ Shibaura Institute of Technology, 307 Fukasaku, Minuma-ku, Saitama 337-8570, Japan \\ E-mail: voshidaeshibaura-it.ac.jp \\ Yuji Motokawa \\ Shibaura Institute of Technology, 307 Fukasaku, Minuma-ku, Saitama 337-8570, Japan \\ E-mail: mf12058dshibaura-it.ac.jp
}

\begin{abstract}
Symmetric and triangle-shaped flux variability in X-ray and gamma-ray light curves has been observed in many blazars. In this work, we carried out a statistical approach to X-ray and gammaray variability characteristics of flares in gamma-ray emitting blazars using archive data. Our results suggest that the time scale of the rise time in flares is equal to that of the decay time. Our results also suggest that the harder flare spectra show the larger spectral variability for the $\mathrm{X}$-ray and gamma-ray flares. In this proceeding, we also discuss a simple model that represent the characteristic X-ray and gamma-ray variability: nearly equal the rise time and decay time, the harder spectral change with the increasing flux, and the larger flare spectral change with the harder spectrum.
\end{abstract}

The 34th International Cosmic Ray Conference,

30 July- 6 August, 2015

The Hague, The Netherlands

${ }^{*}$ Speaker. 


\section{Introduction}

One of the main characteristic of blazars is their rapid and large amplitude variability, which has been observed in most wavelength. As seen in blazar light curves, the variability is stochastic and there is no consistent quiescent flux level. Among those variability, some variability shows time symmetric and triangle-shaped flux variability in the light curves.

J.Kataoka (2000) [四] investigated four TeV blazars of Mrk 421, Mrk501, PKS 2155-304, and 1ES $2344+514$ observed by ASCA and RXTE. He found that the characteristic time scales of individual flares are similar for all $\mathrm{TeV}$ blazars $(\sim 1$ day). He detected the general trend that the amplitude of variation becomes larger at increasing photon energy. He also found that time profiles of each flare ware almost symmetric, meaning that they are characterized by nearly equal rise time and decay time. A.Abdo et al. (2010) [[] presented gamma-ray light curves as well as the first systematic characterization of variability of the 106 blazars observed by Fermi-LAT. The study of temporal profiles of well-resolved flares observed in the 10 brightest Fermi-LAT sources indicates that they generally have symmetric profiles. K.Nalewajko (2013) [3] also presented the systematic study of bright gamma-ray flares in blazars. He selected flares using a very simple and practical definition of a flare as a period of time, during which the flux is above half of the given peak flux. He analyzed 40 brightest gamma-ray flares observed by Fermi-LAT, and derived the light curves and photon index variations.

It is usually believed that the X-ray emission of many blazars arises as synchrotron emission of electrons accelerated at a shock front moving down a jet, and that the gamma-ray emission is due to inverse Compton scattering by the electrons. However, there are no no conclusive models which represent the characteristic X-ray and gamma-ray variability with the rise time nearly equal to the decay time. In this paper, we perform a statistical approach to investigate the X-ray and gamma-ray flares. We also discuss a simple model to give a description the characteristic X-ray and gamma-ray flare in blazars.

\section{Data Analysis}

For X-ray flares in gamma-ray emitting blazars, we used archive data observed by MAXI on International Space Station (ISS) [䧃]. In this analysis, 1 time bin of the light curves is the one orbit period of ISS $(90 \mathrm{~min})$. For the gamma-ray flares, we used the results of the light curves with 1 time bin of 1 day and photon index variations derived by K.Nalewajko (2013) [B]].

Due to the stochastic nature of blazars variability and no consistent quiescence flux level, it is difficult to distinguish between flares and quiescence. In order to define 'flare', A.Abdo et al. (2010) [వ] proposed fitting a function to reproduce a time profile of a single flare. K.Nalewajko (2013) [3] also proposed defining a flare as a period of time, during which the flux is above half of a given peak flux. In order to define 'flare', we propose the other very simple flare definition. Here, we derived the mode value by splitting the maximum flux for each blazar into 50 parts. The threshold level is defined as the mode value of the fluxes for each blazar. A flare is defined as a contiguous period of time, during which the flux exceeds the threshold level with $3 \sigma$ level. This threshold level is attained twice at the beginning and the end of the flare. A flare may include sub- 
peaks, but they are treated as one flare. This definition does not require the peak flux in advance, and the flares tend to have longer duration periods than that of K.Nalewajko (2013).

By using this definition, we picked out the flares from the blazar's light curves. From the flares, we derived the flare peak $F_{\text {peak }}$, the flare rise time $T_{r}$, the flare decay time $T_{d}$, the flare duration $T_{F l}=T_{r}+T_{d}$, the flare asymmetry parameter $k=\left(T_{d}-T_{r}\right) /\left(T_{d}+T_{r}\right)$, and the flare net count $F N C$ which is the integrated flux during the flare above the threshold. For the X-ray flares observed by MAXI, we also derived the average hardness ratio in a flare $\langle H R\rangle$ and the root mean square value of hardness ratios in a flare $r m s(H R)$. The hardness ratio is a ratio of $10-20 \mathrm{keV}$ count rate to $4.0-10 \mathrm{keV}$ count rate. For the gamma-ray flares, in addition to $F_{p e a k}, T_{r}, T_{d}, T_{F l}=T_{r}+T_{d}$, and $k$, we also derived the average photon index $\langle\Gamma\rangle$ in a flare and the photon index scatter r.m.s. $r m s(\Gamma)$ in a flare, as described in K.Nalewajko (2013) [B]].

\section{Results}

We derived a sample of $75 \mathrm{X}$-ray flares from 3 gamma-ray emitting blazars observed by MAXI. For details, the sample is 33 flares of Mrk 421, 41 flares of Mrk 501, and 1 flare of PKS 2155-304. We also derived a sample of 36 gamma-ray flares from 6 blazars observed by Fermi-LAT. For details, the sample is 7 flares of 3C 273, 10 flares of 3C 454.3, 1 flare of PKS 0401-362, 10 flares of PKS 1222+216, 1 flare of PKS 1329-049, and 7 flares of PKS 1510-089. Table $\square$ and Table $\square$ show summaries of the X-ray flares and gamma-ray flares of the gamma-ray emitting blazars.

Table 1: Summary of the X-ray flares of gamma-ray emitting blazars observed by MAXI

\begin{tabular}{lcccccc}
\hline Source & $\begin{array}{c}\text { No. of } \\
\text { flare }\end{array}$ & $\begin{array}{c}\text { Average } \\
\overline{T_{F l}}(\mathrm{~d})\end{array}$ & $\begin{array}{c}\text { Average } \\
\bar{k}\end{array}$ & $\begin{array}{c}\text { Average } \\
F_{\text {peak }}(\mathrm{c} / \mathrm{s})\end{array}$ & $\begin{array}{c}\text { Average } \\
\overline{\langle H R\rangle}\end{array}$ & $\begin{array}{c}\text { Average } \\
\overline{F N C}(\mathrm{c})\end{array}$ \\
\hline Mrk 421 & 33 & 0.833 & 0.024 & 0.312 & 0.49 & $1.27 \times 10^{4}$ \\
Mrk 501 & 41 & 0.377 & -0.11 & 0.366 & 2.22 & $6.62 \times 10^{3}$ \\
PKS 2155-304 & 1 & 0.188 & 0.333 & 5.22 & 0.497 & $3.75 \times 10^{4}$ \\
\hline
\end{tabular}

Table 2: Summary of the gamma-ray flares of blazars observed by Fermi-LAT

\begin{tabular}{lcccccc}
\hline Source & $\begin{array}{c}\text { No. of } \\
\text { flare }\end{array}$ & $\begin{array}{c}\text { Average } \\
\overline{T_{F l}}(\mathrm{~d})\end{array}$ & $\begin{array}{c}\text { Average } \\
\bar{k}\end{array}$ & $\begin{array}{c}\text { Average } \\
F_{\text {peak }}\left(\mathrm{ph} / \mathrm{cm}^{2} / \mathrm{s}\right)\end{array}$ & $\begin{array}{c}\text { Average } \\
\overline{\langle\Gamma\rangle}\end{array}$ & $\begin{array}{c}\text { Average } \\
\overline{F N C}\left(\mathrm{ph} / \mathrm{cm}^{2}\right)\end{array}$ \\
\hline 3C 273 & 7 & 2.43 & 0.048 & $6.08 \times 10^{-6}$ & -2.42 & 3.25 \\
3C 454.3 & 10 & 14.5 & 0.063 & $1.56 \times 10^{-5}$ & -2.41 & 52.9 \\
PKS 0402-362 & 1 & 4.40 & 0.000 & $6.79 \times 10^{-6}$ & -2.28 & 6.56 \\
PKS 1222+216 & 10 & 4.16 & 0.069 & $7.79 \times 10^{-6}$ & -2.17 & 3.94 \\
PKS 1329-049 & 1 & 3.00 & -0.200 & $4.66 \times 10^{-6}$ & -2.48 & 2.97 \\
PKS 1510-089 & 7 & 9.66 & 0.034 & $8.64 \times 10^{-6}$ & -2.27 & 14.2 \\
\hline
\end{tabular}

Figure $\square$ presents scatter plots between the rise time and the decay time from the flares ob-

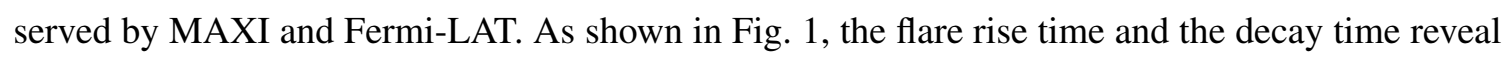


a correlation with a correlation coefficient of 0.49 for the X-ray flares and a correlation coefficient of 0.91 for the gamma-ray flares. Figure $\square$ also presents distributions of the time asymmetry parameter $k$ of the flares. The value of $k$ becomes positive in the case of the decay time larger than the rise time and vice versa, spanning between -1 and 1. As shown in Fig. \, although the decay time in the gamma-ray flares trends to be comparatively larger time than the rise time, the time scales of the decay time in the $\mathrm{X}$-ray and gamma-ray flares are equal to those of the rise time. The average value and the root mean square of $k$ in the X-ray flares are -0.045 and 0.466 . The average value and the root mean square of $k$ in the gamma-ray flares are 0.047 and 0.419 , respectively.
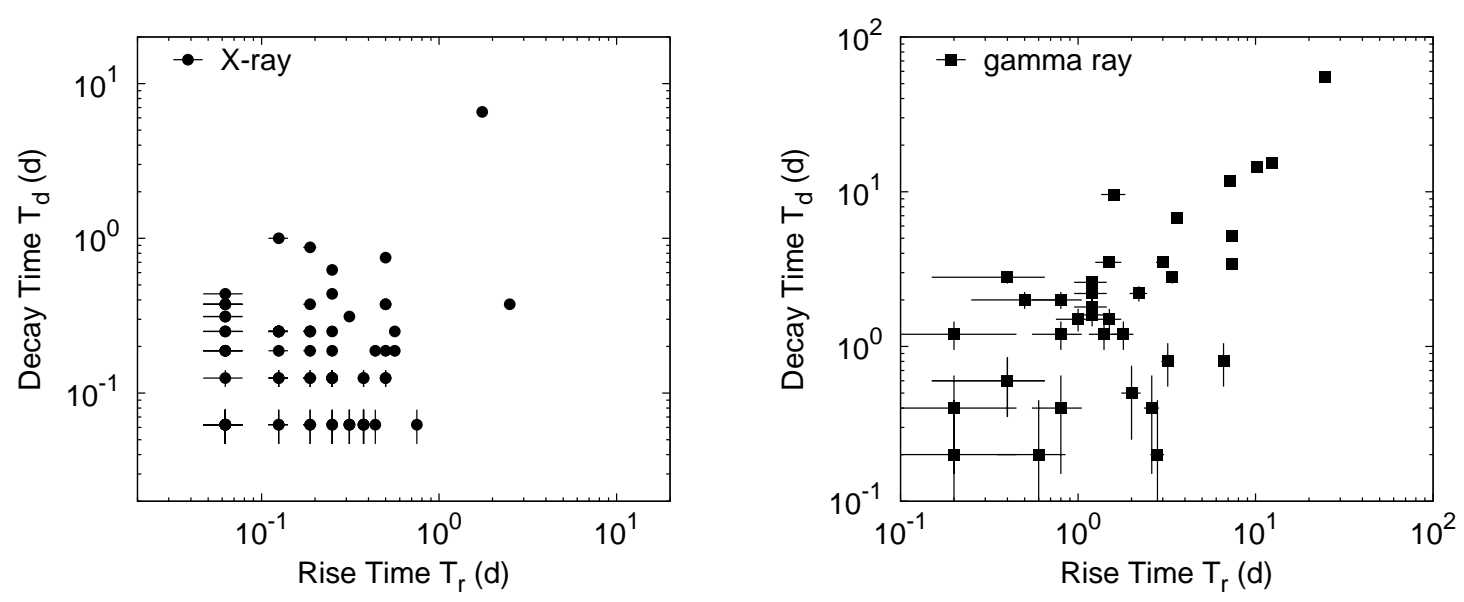

Figure 1: Scatter plots between the rise time and the decay time from the flares observed by MAXI (left panel) and Fermi-LAT (right panel).

Figure [3] presents a scatter plot between the mean hardness ratio $\langle H R\rangle$ and the hardness ratio scatter root mean square $r m s(H R)$ from the flares observed by MAXI, and a scatter plot between the mean photon index $\langle\Gamma\rangle$ and the photon index scatter root mean square $r m s(\Gamma)$ from the flares observed by Fermi-LAT. As shown in Fig. [3, the mean hardness ratio $\langle H R\rangle$ and the hardness ratio scatter $r m s(H R)$ for the $\mathrm{X}$-ray flares reveal a correlation with a correlation coefficient of 0.64 . The mean photon index $\langle\Gamma\rangle$ and the photon index scatter $\operatorname{rms}(\Gamma)$ for the gamma-ray flares reveal a correlation with a correlation coefficient of 0.27 .

\section{Discussion}

We discuss a simple model [5] to give a description these flare behaviors as presented in the previous section. This model is based on a model given by F.Makino (2000) [四]. In this model, we use a kinetic equation of the spectrum of accelerating and emitting electrons, expressed by

$$
\frac{d N_{e}}{d t}+\frac{\partial}{\partial E}\left(g(E) N_{e}\right)+\frac{N_{e}}{T(E)}=Q(E, t), \quad g(E)=\beta E-b E^{2}
$$



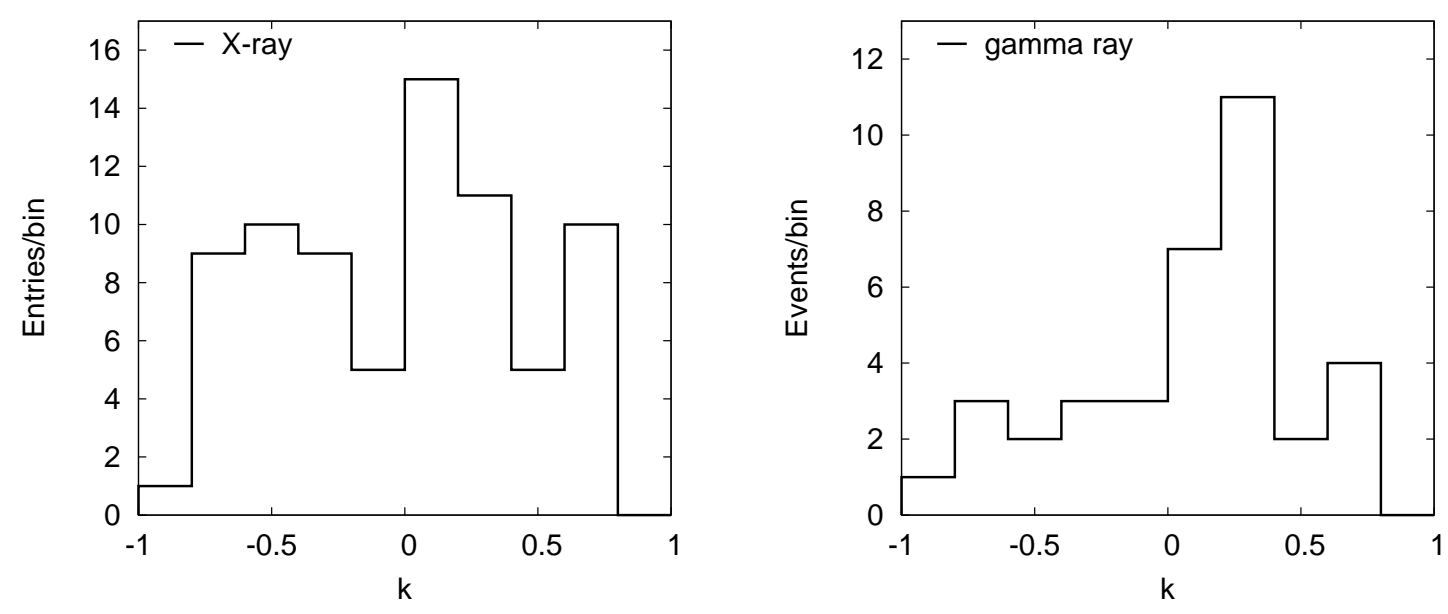

Figure 2: Distributions of the time asymmetry parameter $k$ of the flares observed by MAXI (left panel) and Fermi-LAT (right panel).

where $E$ is the electron energy, $t$ is the time, $\beta$ is the acceleration efficiency, $b$ is the coefficient of energy loss, $T$ is the escape time, and $Q(E, t)$ is the injection rate. The general solution of this equation is given by F.Makino et al. (1996) [四] and F.Makino (2000) [四].

We propose that the injection rate $Q$ of low energy electrons increases linearly in the rise phase and decreases linearly in the decay phase in the following,

$$
\begin{aligned}
& Q(E, t)=K \delta\left(E-E_{0}\right) t \quad\left(0<t<t_{\text {cros }} / 2\right) \\
& Q(E, t)=K \delta\left(E-E_{0}\right)\left(t_{\text {cros }}-t\right) \quad\left(t_{\text {cros }} / 2<t<t_{\text {cros }}\right)
\end{aligned}
$$

where $K$ is the normalization factor, $E_{0}$ is the energy of injected electrons and $t_{\text {cros }}$ is the duration time of the rise to decay phase. For the rise phase, the electron spectrum is given by

$$
N_{e}=K\left(\frac{\beta-b E}{\beta-b E_{0}}\right)^{\frac{1}{\beta T}-1}\left(\frac{E}{E_{0}}\right)^{-\frac{1}{\beta T}-1}\left(t-\frac{1}{\beta} \log \frac{E}{E_{0}}+\frac{1}{\beta} \log \frac{\beta-b E}{\beta-b E_{0}}\right) .
$$

In a similar way, the electron spectrum in the decay phase is derived.

In this work, we converted from the electron spectrum $N_{e}(E, t)$ to the X-ray spectrum $N_{X}(E, t)$ by using a monochromatic approximation, although the monochromatic approximation might inappropriate for the gamma-ray spectrum $N_{\gamma}(E, t)$. This model can represent the characteristic X-ray flare variability as described in [[] in the following.

1. The X-ray flux increases linearly with time in the rise of the flare and decreases linearly in the decay,

2. The rise time of the flare is equal to the decay time.

3. The spectrum becomes harder in the rise and softer in the decay. 

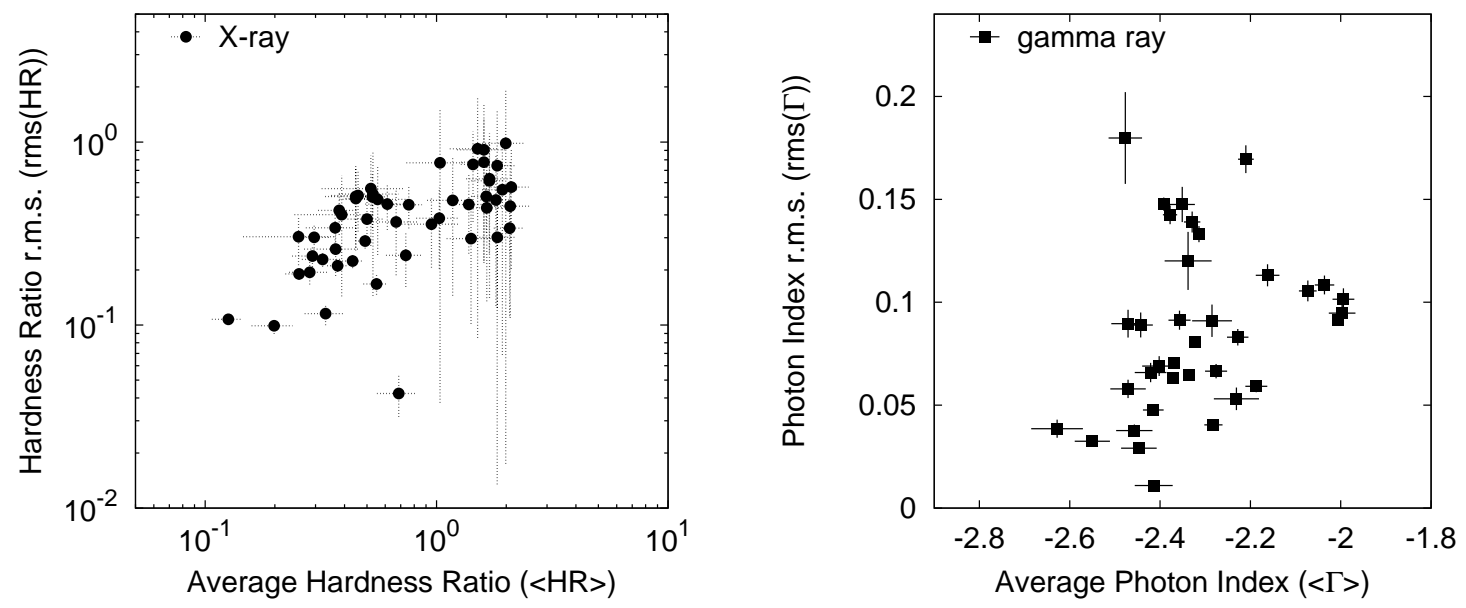

Figure 3: Scatter plot between the average hardness ratio $\langle H R\rangle$ and the hardness ratio scatter root mean square $\operatorname{rms}(H R)$ from the flares observed by MAXI (left panel), and scatter plot between the mean photon index $\langle\Gamma\rangle$ and the photon index scatter root mean square $r m s(\Gamma)$ from the flares observed by Fermi-LAT (right panel).

In the internal shock scenario (e.g. [[]]), X-ray and gamma-ray flares occur through shock formation in the collisions of faster blobs with slower blobs in jets. In the crossover region of a faster blob with a slower blob, low energy electrons are injected, leading to acceleration to high energy electrons and generation of synchrotron X-ray emission. Corresponding to the crossover region size, the injection rate of low energy electrons might increase linearly in the catching up phase and decrease linearly in the passing phase.

We calculated the time variations of the X-ray flares observed by MAXI by using this model. Figure 4 presents an example of the calculated $X$-ray hardness ratio $(10-20 \mathrm{keV} / 4-10 \mathrm{keV})$ vs. the $\mathrm{X}$-ray flux. Here, the magnetic field strength $B$ is $0.1 \mathrm{G}, E_{0}$ is $1 \mathrm{MeV}, \beta$ is $3 \times 10^{-5} \mathrm{~s}^{-1}, T$ is

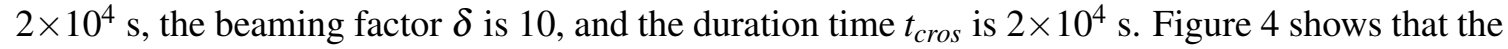
$\mathrm{X}$-ray spectra become harder in the rise and softer in the decay phase. This X-ray spectral variation has been observed in the X-ray flares of many blazars (e.g. [9]).

Figure $[$ presents the calculated scatter plot between the average hardness ratio $\langle H R\rangle$ and the root mean square value of hardness ratios $r m s(H R)$ in an X-ray flare. We calculated the scatter plots with 254 different parameter sets. Here, the magnetic field strength $B$ ranges from $1 \times 10^{-3} \mathrm{G}$ to $0.12 \mathrm{G}, \beta$ ranges from $1.2 \times 10^{-5} \mathrm{~s}^{-1}$ to $6 \times 10^{-4} \mathrm{~s}^{-1}$, and $T$ ranges from $5 \times 10^{3} \mathrm{~s}$ to $5 \times 10^{5} \mathrm{~s}$. The other parameters are fixed as follows: $E_{0}=1 \mathrm{MeV}, \delta=10$, and $t_{\text {cros }}=2 \times 10^{4} \mathrm{~s}$. As shown in Fig. [1, the observed scatter plots suggest that the harder flare spectra show the larger spectral variability for the X-ray and gamma-ray flares. The calculated result in Fig. [ shows the similar behavior with the observed result in Fig. [3. In this model, a strong correlation between $\langle H R\rangle$ and $r m s(H R)$ appears in the change of the escape time $T$ and the acceleration efficiency $\beta$. The 
magnetic field $B$ contributes in a small fraction and causes the decrease of $r m s(H R)$ in the high average hardness ratio $\langle H R\rangle$.

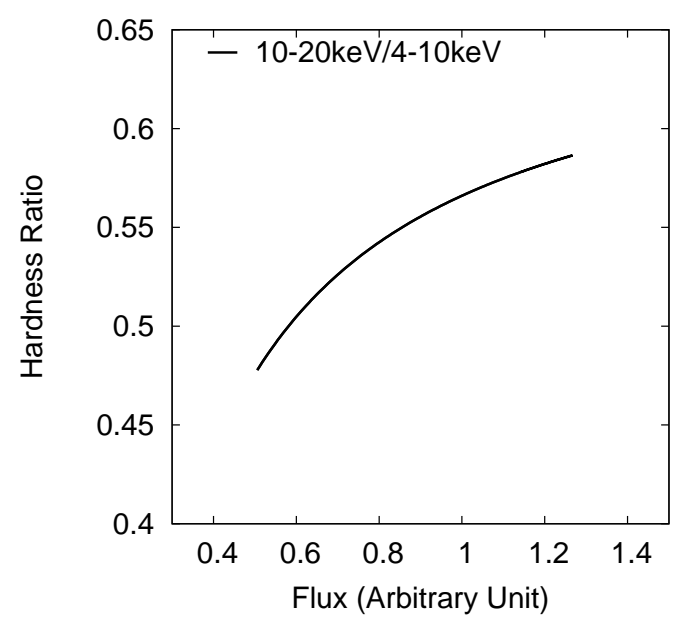

Figure 4: An example of the calculated X-ray hardness ratio $(10-20 \mathrm{keV} / 4-10 \mathrm{keV})$ vs. the X-ray flux. See text for details.

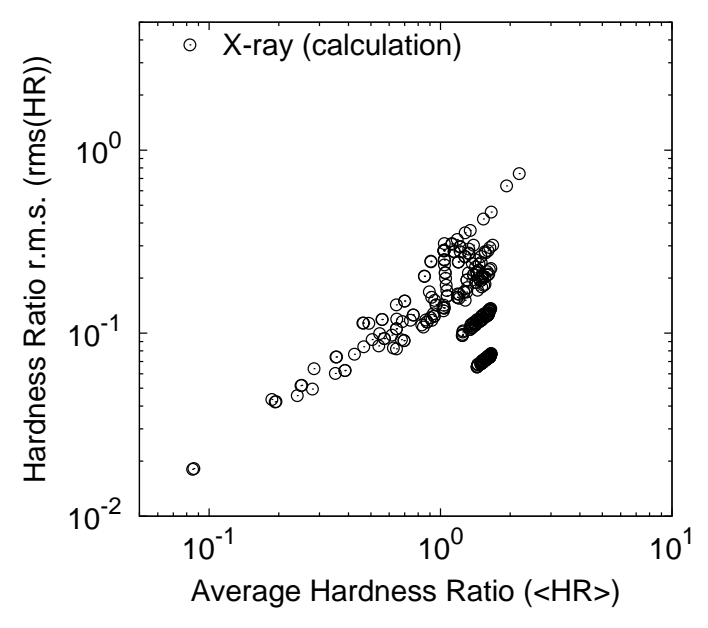

Figure 5: Calculated scatter plot between the average hardness ratio and the hardness ratio scatter root mean square. See text for details.

\section{Conclusion}

This statistical approach suggests that the time scale of the rise time is equal to that of the decay time for the X-ray and gamma-ray flares in gamma-ray emitting blazars. This approach also suggests that the harder flare spectra show the larger spectral variability for the X-ray and gammaray flares in gamma-ray emitting blazars. These results can be represented by a simple model based on F.Makino (2000) [ [ $]$ ]. In the future paper, we will calculate the gamma-ray flare variability and compare the calculated results with the observed data.

We are sincerely grateful to Dr. K.Nalewajko for kindly providing us the analysis results of gamma-ray emitting blazars.

\section{References}

[1] J.Kataoka, $\mathrm{PhD}$ thesis, 2001.

[2] A.A.Abdo et al., Astrophys. J. 722, 520-542, 2010.

[3] K.Nalewajko, Mon. Not. R. Astron. Soc. 430, 1324-1333, 2013.

[4] MAXI RIKEN http://maxi.riken.jp/

[5] K.Yoshida, Proc. of IAU Symposium 313, 97, 2014. 
[6] F.Makino et al., MPE Report 263, 413-416, 1996.

[7] F.Makino, Adv. Space Res. 25, 723-728, 2000.

[8] M.Spada et al., Mon. Not. R. Astron. Soc. 325, 1959-1570, 2001.

[9] L.Marashi et al., Adv. Space Res. 25, 713, 2000. 